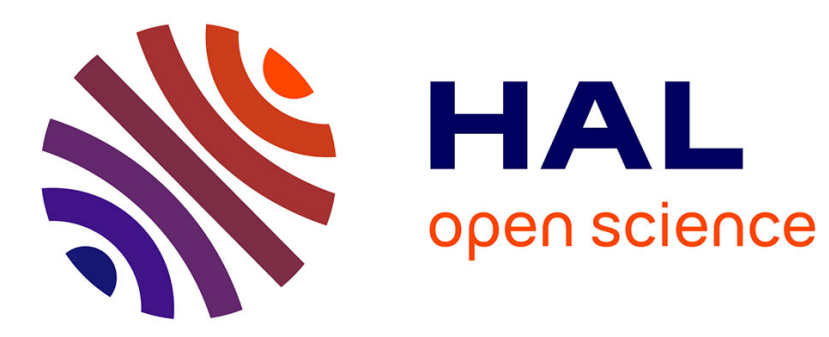

\title{
De la représentation de la terre à sa reproduction : l'invention des géoramas au dix-neuvième siècle
}

\author{
Jean-Marc Besse
}

\section{To cite this version:}

Jean-Marc Besse. De la représentation de la terre à sa reproduction : l'invention des géoramas au dixneuvième siècle. I. Laboulais. Combler les blancs de la carte. Modalités et enjeux de la construction des savoirs géographiques (XVIIe-XIXe siècle), Presses Universitaires de Strasbourg, pp.34-59, 2004. halshs-00113283

\section{HAL Id: halshs-00113283 \\ https://shs.hal.science/halshs-00113283}

Submitted on 12 Nov 2006

HAL is a multi-disciplinary open access archive for the deposit and dissemination of scientific research documents, whether they are published or not. The documents may come from teaching and research institutions in France or abroad, or from public or private research centers.
L'archive ouverte pluridisciplinaire HAL, est destinée au dépôt et à la diffusion de documents scientifiques de niveau recherche, publiés ou non, émanant des établissements d'enseignement et de recherche français ou étrangers, des laboratoires publics ou privés. 
I. Laboulais (dir.), Combler les blancs de la carte. Modalités et enjeux de la construction des savoirs géographiques (XVII ${ }^{e}$ XIX $X^{e}$ siècle), Presses Universitaires de Strasbourg, 2004, p. 34-59.

\section{De la représentation de la terre à sa reproduction : l'invention des géoramas au dix-neuvième siècle.}

\section{1 - L’imagination géographique}

La carte est-elle le meilleur moyen pour représenter le territoire ? La question est classique, et l'on a beaucoup écrit, ces dernières années, sur les conceptions positivistes et naturalistes de la carte, pour dénoncer ce fantasme de l'exactitude qui serait, au mieux, l'expression d'une naïveté, et au pire celle d'une volonté de dissimulation. Faut-il en conclure, de façon polémique, que toute carte est structurellement fausse ? Ou bien faut-il rappeler, après Nelson Goodman, que c'est par nature que la carte n'est pas le territoire, qu'elle est schématique, sélective et conventionnelle par vocation et que c'est même ce qui constitue sa vertu principale ? Les blancs de la carte, qu'ils soient voulus ou subis, fabriqués ou regrettés, ne seraient, en ce sens, rien d'autre que les symptômes de cet écart constitutif de l'opération cartographique elle-même.

Mais la question de la représentation cartographique n'est pas seulement une question de vérité. En d'autres termes, la question des blancs de la carte ne se pose pas seulement dans la perspective des relations entre carte et territoire, et dans une orientation référentielle. On voudrait évoquer, ici, une autre orientation, qu'on dira pragmatique et psychologique, dans la mesure où elle concerne non pas tant la valeur représentative intrinsèque de la carte que son efficacité ou sa force représentative dans l'esprit de ceux qui la regardent. En d'autres termes, la question est moins celle de la vérité de la carte que celle des moyens pragmatiques qu'elle met en œuvre pour que les spectateurs saisissent intellectuellement et perceptivement cette vérité et s'en pénètrent.

En quoi la carte fait-elle réalité, si l'on peut dire, aux yeux de ceux qui la regardent ? Comment la carte rend-elle présente au spectateur la réalité qu'elle lui désigne? Quelle conscience de l'espace la carte permet-elle d'installer dans l'esprit du spectateur? 
Ces questions relèvent moins du domaine de l'épistémologie historique que de celui de la psychologie historique : elles concernent moins le progrès des connaissances géographiques que celui de l'installation d'une « conscience géographique » dans la culture d'une époque et du progrès de cette « conscience ». Les pédagogues de la géographie ont rencontré depuis longtemps ce problème, et lui ont donné un nom : c'est celui de l'imagination géographique.

Le pédagogue jésuite Jean François, dans son ouvrage La science de la géographie (Rennes, 1652), distingue quatre degrés dans les apprentissages géographiques : l'intelligence des termes, l'usage des cartes, la représentation des réalités géographiques « sur le Globe naturel en leur lieu propre, et en leur assiette naturelle » ${ }^{1}$, et le raisonnement explicatif proprement dit. De ces quatre degrés, qui correspondent en même temps à une topographie des facultés psychologiques, le troisième, qui concerne l'imagination, est le plus difficile à acquérir, selon Jean François. Il est pourtant nécessaire, car une imagination « bien formée » prépare l'esprit à consentir aux vérités de la raison ${ }^{2}$. Par conséquent, c'est sur le plan de l'imagination, et grâce à l'opération de l'imagination, que les termes géographiques et les signes cartographiques acquièrent un sens pour ceux qui les manipulent.

L'imagination dont Jean François souligne ici le caractère incontournable se caractérise essentiellement par deux propriétés, corrélatives et symétriques. Elle est d'une part, un mouvement intentionnel de l'esprit, qui porte celui-ci depuis la représentation vers la réalité, mouvement qui permet à l'esprit, pour reprendre le vocabulaire du pédagogue jésuite, de passer de la carte et du « globe artificiel » vers le « globe naturel ». L'imagination ne consiste pas ici à faire venir le réel dans une image, mais, par un mouvement strictement inverse, à transporter l'esprit depuis l'image vers le réel, par une sorte d'incarnation du signe cartographique devant la conscience. Ce «transport» est rendu possible par la mise en œuvre de ce que Jean François appelle la «similitude », c'est-à-dire par la capacité de l'esprit à passer du petit au grand et du grand au petit, à parcourir les échelles spatiales, à concevoir l'analogie des figures ressemblantes quelles que soient leurs tailles respectives. C'est la similitude qui fait comprendre la relation entre le «globe artificiel » et le «globe naturel », et c'est le parcours de cette similitude que François désigne sous le nom d'imagination. Mais, d'autre part, l'imagination est aussi une puissance figurative. Elle correspond au pouvoir que possède l'esprit de mettre la réalité en images et de raisonner à

1 J. François, La science de la géographie, Rennes, 1652, p. 36.

2 Ibid. 
partir de ces supports visuels. La forme de rationalité mise en œuvre par le géographe est, en fait, assez proche de celle de l'architecte, qui «fait voir en un petit modèle un ample palais que quelque riche monarque aura dessein de bâtir ${ }^{3}$ ». La géographie est un savoir dont les raisonnements s'appuient sur la vision et sur l'image, et où, plus précisément, les raisonnements prennent la forme de mouvements de l'œil et de l'imagination. C'est un savoir de l'analogie.

La question «pédagogique » consiste alors à favoriser l'usage de cette imagination géographique, seule capable d'installer dans la conscience la grandeur véritable des réalités géographiques. Il s'agit par conséquent de réunir les moyens et les supports pédagogiques, c'est-à-dire les espaces figuratifs, propres au développement de cette imagination. La position de Jean François est sur ce point assez radicale par la diversité même des supports qu'il préconise :

Les matières que l'on choisit plus ordinairement sont celles, qui sont plus propres à recevoir facilement les lignes d'un crayon, les traits d'une plume, les couleurs d'un pinceau, la gravure des burins, et l'impression des planches gravées: comme certes on en voit maintenant en telle multitude et variété, qu'on n'y peut presque rien souhaiter davantage. Les plus rares, et extraordinaires seraient les tapisseries, les lambris, et les parois, et autres semblables sujets ; et même les parterres dans les jardins, auxquels on pourrait facilement donner la figure d'une Carte, et mettre en eau effective la partie, qui représenterait la mer: comme l'autre serait de terre avec les élévations, et abaissements conformes à ceux du Globe, pour donner la pente aux Rivières, et le moyen de couler depuis leurs sources jusques à la mer. Les pavés dans les salles sont ici très propres : car il n'est pas plus difficile de faire peindre les carreaux en couleur de mer ou autre, selon que le sujet le demandera, pour faire des Cartes ; que pour d'autres figures, et étant peints les faire cuire, et étant cuits les appliquer avec l'ordre requis, pour représenter une Province, et même les deux côtés parallèles de chaque carreau montreraient les longitudes, les deux autres les latitudes, et leur longueur contiendrait /349/ un degré, une minute ou quelque autre partie de ces dimensions. Et comme l'espace est plus grand, on y verrait plus distinctement les parties d'une Province, et par cette sorte on s'instruirait insensiblement à la Géographie ; et en se promenant on parcourrait bientôt un pays de grande étendue. Les voûtes sphériques concaves sont propres à recevoir la représentation de la moitié du Globe. ${ }^{4}$

$3 \quad$ Ibid., p. 346.

4 Ibid., pp. 348-349. 
Cette profusion des « matières » envisagées par Jean François (décorations murales, tapisseries, voûtes peintes, sols, maquettes, jardins) ne doit pas abuser : il s'agit bien, à chaque fois, de créer des espaces qui ont un double caractère. Ils sont à la fois des représentations, des images d'un territoire plus ou moins étendu, et des territoires euxmêmes plus ou moins grands. Ils sont à la fois réels et imaginaires. Ce sont des espaces de représentation, des espaces ambigus où l'imagination et la réalité se superposent et parfois se confondent. Mais ce qu'il faut souligner c'est qu'ils sont avant tout des espaces d'intensification. Intensification de l'imagination et de la conscience géographiques, qui par l'intermédiaire de ces espaces, au sein de ces espaces, accomplissent véritablement leur vocation : celle de rendre sensible le spectateur à l'ampleur concrète des réalités terrestres.

L'histoire «matérielle » de la géographie (c'est-à-dire, ici, l'histoire de sa diffusion dans le public) a rencontré à plusieurs reprises les perspectives dessinées par le pédagogue jésuite. Depuis la Renaissance, la géographie est devenue un élément important dans la définition des cultures visuelles modernes, européennes notamment. On ne compte plus les cérémonies publiques, les fêtes, les défilés, les programmes décoratifs, les créations artistiques, les jardins mêmes, qui ont pris comme thème ou comme support les représentations du monde que leur livrait la géographie ${ }^{5}$. Au début du dix-neuvième siècle, ces espaces de l'imagination géographique ont donné lieu à l'apparition, à Paris d'abord, sur le Boulevard, d'une forme originale, le géorama. Le géorama est un bâtiment qui, lorsqu'il est créé, relève à la fois de la salle de cours et de la salle d'exposition. Destiné à la présentation des résultats de la science, il est en même temps, par sa simple situation dans la topographie des spectacles métropolitains, représentatif des formes nouvelles que prend la culture géographique au moment où apparaît le « grand public ».

\section{2 - La géographie dans la ville : la diffusion des panoramas}

En 1817, dans son compte rendu du Salon, le critique d'art français Edme-François Miel propose l'édification, par le gouvernement, de «panoramas permanents » et, surtout, leur distribution raisonnée à l'intérieur de Paris. Ces panoramas permanents seraient, indique-t-il,

commodes pour les Parisiens, qui généralement aiment à voyager sans sortir de chez eux; pour la jeunesse, ils deviendraient classiques. En visitant ces véridiques rotondes, les jeunes gens

5 Cf., pour une histoire de quelques-uns de ces dispositifs, J.-M. Besse, Face au monde. Atlas, jardins, géoramas, Paris, Desclée de Brouwer, 2002. 
complèteraient leur instruction géographique et rectifieraient sur les lieux [...] beaucoup de fausses notions puisées dans les livres. Un ordre systématique pourrait présider à l'arrangement de cet univers. Les places de commerce les plus importantes, Lyon, Bordeaux, Marseille, Londres, Amsterdam, Philadelphie, seraient rangées dans le quartier de la Bourse et des affaires commerciales ; les villes qui ont leur plus grande importance comme capitale, comme centres des relations diplomatiques de l'Europe, Pétersbourg, Vienne, Berlin, Madrid, Constantinople, seraient placées à proximité de l'hôtel des affaires étrangères ; les plus célèbres champs de bataille, Marengo, Iéna, Austerlitz, Wagram, seraient le plus digne voisinage des Invalides, et les guerriers mutilés pour la patrie reverraient sans péril, mais non sans orgueil, les théâtres de leur gloire. Près du Jardin du Roi, on verrait les villes d'Orient et celles des Tropiques, qui, en offrant aux spectateurs d'autres hommes, d'autres animaux, une autre végétation, sembleraient mettre en scène les galeries d'histoire naturelle, et donneraient la meilleure leçon de cette science attrayante. Les lieux qui empruntent des arts une grande partie de leur célébrité, Naples, Rome, Florence, Venise, Athènes, occuperaient les environs de ce Louvre, qui leur doit ses ornements et ses chefs-d'œuvre. ${ }^{6}$

La ville de Paris n'est pas représentée dans les panoramas que suggère Miel. Sans doute parce qu'elle en est le cadre de réception, le support spatial. Mais la conséquence est saisissante : le territoire parisien deviendrait, dans cette perspective, un gigantesque musée du monde entier, qui se donnerait à voir sous ses aspects les plus significatifs, dans des lieux qui en sont comme des résumés. C'est une étrange expérience urbaine qui ainsi se dessine dans la proposition de Miel : dans le quartier de la Bourse, la représentation des autres places commerciales ; près du Jardin du Roi, le spectacle des lieux de l'exotisme savant ; au Louvre, l'évocation des sites où les œuvres exposées dans le musée ont leur origine. A chaque fois, en un certain sens, le panorama met en scène et renforce ce dont il s'agit dans la partie de la ville de Paris où se trouve, effectivement, le visiteur. Le panorama, dans lequel se trouve ce dernier, fournit, si l'on peut dire, une signification et une profondeur à l'endroit de la ville où ce panorama est placé. Ainsi, le quartier de la Bourse, comme partie de l'espace urbain, est justifié dans son existence par la relation que le panorama exhibe entre ce quartier et d'autres places, qui en Europe ont une vocation équivalente. De même, le jardin du Roi est renforcé et fondé dans sa présence urbaine par l'exposition des sites d'où proviennent, justement, les objets et les échantillons de nature qu'il présente au public. Ces fonctions de la ville, qui sont en même temps des lieux de vie et de sociabilité, reçoivent

6 E.F. Miel, Essai sur les beaux-arts, et particulièrement sur le salon de 1817, ou examen critique des principaux ouvrages d'art exposés dans le cours de cette année, Paris, 1817, pp. 351-352. 
ainsi, par l'intermédiaire des panoramas, une « référence », et par conséquent une sorte de vérité. Le panorama géographique met en place, peut-on en conclure, une expérience urbaine qui est caractérisée par la réflexivité. Le panorama parle au spectateur à la fois des autres endroits du monde et de l'endroit de la ville où il se trouve actuellement. Singulière expérience, qui consiste à marcher dans la ville comme dans un vaste espace voué au spectacle du monde, à marcher dans une sorte de topographie savante, et à stationner, à intervalles réguliers, dans des lieux où l'expérience urbaine elle-même s'intensifie. C'est la ville elle-même qui ainsi s'expose, comme lieu de spectacle, et se raconte, à ceux qui la fréquentent .

Le rêve urbain de Miel s'appuie sur une réalisation qui est considérée comme typique du dix-neuvième siècle dans les métropoles occidentales: le panorama, dont on connaît l'importance dans la redéfinition de la topographie urbaine des spectacles, en particulier à Paris, au moment de la naissance des Grands Boulevards ${ }^{7}$. On sait que l'idée initiale du panorama est due à un peintre irlandais, Robert Barker. Celui-ci dépose un brevet en 1787 pour une invention qu'il nomme La Nature à coup d'œeil, destinée à « présenter des vues de la nature en grand format par la peinture à l'huile, la fresque, les couleurs à l'eau, les crayons ou n'importe quelle autre façon de peindre ou de dessiner ${ }^{8}$ ». Dès l'origine, semblet-il, les deux principes du dispositif panoramique sont établis : le panorama est un espace circulaire et clos, qui ouvre à la contemplation sans limites de la totalité du monde. Le procédé est introduit à Paris en1799 par l'américain Robert Fulton, qui, ayant obtenu un brevet d'importation, fait édifier une rotonde à l'intérieur du jardin des Capucines (dans l'enceinte délimitée par le boulevard des Capucines et la rue Louis-le-Grand). Le site de l'ancien couvent des Capucines est devenu, au début du dix-neuvième siècle, un des lieux de plaisir les plus fréquentés de la capitale. Dans les ruines de l'église, du cloître et du réfectoire, se sont installés « des échoppes de marchands de gâteaux, de jouets d'enfants et d'orviétan ; des physiciens ambulants, des danseurs de corde, des lanternes magiques et des baraques où l'on montrait des phénomènes et des animaux vivants ${ }^{9}$ ». Le panorama est ouvert en septembre, avec une Vue de Paris depuis les Tuileries, réalisée par Pierre Prévost. Deux nouvelles rotondes sont construites boulevard Montmartre vers 1801 par James

7 L'histoire de la peinture et de l'architecture des panoramas est maintenant relativement bien connue. Cf. les travaux, entre autres, de S. Oettermann, Das panorama: Die Geschichte eines massenmediums, Francfort, Syndicat, 1980 ; S. Bordini, Storia del panorama. La visione totale nella pittura del XIX secolo, Rome, Offizina Edizioni, 1984 ; B. Comment, Le XIX siècle des panoramas, Paris, Adam Biro, 1993.

8 Brevet anglais de Barker, $\mathrm{n}^{\circ}$ 1612, 19 juin 1787.

9 L. Lurine, Les rues de Paris, t. 1, Paris, 1844, p. 163. 
Thayer, auquel Fulton avait vendu son brevet. Dans ces édifices, devenus rapidement populaires, Prévost, qui s'impose comme le principal panoramiste du temps, présente au public un Panorama de Lyon, puis une Vue d'Amsterdam. Y succèderont des vues de Londres, de Rome, de Naples, exposées avec un succès toujours plus important.

En 1808, Thayer et Prévost, ayant acheté le cirque Franconi, y installent une nouvelle rotonde, à l'angle du boulevard des Capucines et de la rue Neuve-Saint-Augustin (rue Daunou). La taille de ce nouveau bâtiment permet d'y accueillir des toiles de trentedeux mètres de diamètre sur seize mètres de hauteur (les rotondes du boulevard Montmartre n'offraient que quatorze mètres de diamètre). Le panorama est inauguré en 1809 avec une Vue de la rencontre entre les empereurs français et russes à Tilsit. Une Bataille de Wagram est exposée l'année suivante, que visite l'Empereur. Plus tard, Prévost y présentera les panoramas de Jérusalem (1819) et d'Athènes (1821), qui rencontreront un grand succès. L'engouement ne disparaît pas à la mort de Prévost (1823). En 1831, alors que les rotondes du boulevard Montmartre sont détruites, un élève d'Horace Vernet, le Colonel Langlois, ouvre une nouvelle rotonde de trente-huit mètres de diamètre au 14, rue des Marais-duTemple (derrière l'actuelle place de la République), qui est inaugurée par la Bataille navale de Navarin. Langlois introduit plusieurs transformations et perfectionnements dans les procédés d'éclairage (gaz, verres dépolis pour supprimer les ombres sur la toile) et de présentation (un faux-terrain sépare la plate-forme et la toile, des ventilations miment l'incendie), qui en renforcent la puissance d'illusion. Surtout, Langlois présente la plateforme sous l'apparence d'un véritable pont de bateau, auquel les spectateurs accèdent après avoir traversé une série de cabines et de coursives. Cette dernière innovation, destinée à porter l'illusion à son maximum, est reconnue comme un des éléments clés du succès de Langlois. Ce dernier présentera en 1833 une Bataille d'Alger, puis en 1834 une Bataille de la Moskova, elles aussi couronnées de succès. Langlois est alors en mesure de faire édifier une nouvelle rotonde, de quarante mètres de diamètre, sur les Champs-Elysées, entre le Cours de la Reine et le Grand Carré des Fêtes. Le panorama (l'actuel théâtre du RondPoint), édifié par l'architecte Hittorff, est inauguré en 1839 par un Incendie de Moscou observé depuis une des tours du Kremlin. La toile est remplacée, en 1843, par une Bataille d'Eylau, qui assure un succès public définitif à Langlois. Ce dernier ouvrira un autre panorama aux Champs-Elysées, en 1860, et d'autres établissements seront également 
ouverts dans la capitale jusqu'à la fin du dix-neuvième siècle, rencontrant une faveur toujours égale auprès du public ${ }^{10}$.

La description que laisse Hittorff du procédé panoramique permet de mieux comprendre la vigueur de l'effet qu'il produit, qu'on a du mal à imaginer aujourd'hui :

Le nom de panorama, composé de deux mots grecs (pan, tout, et orama, vue, c'est-à-dire vue d'un tout), n'est applicable rationnellement qu'à une peinture proprement dite, qui représente une vue générale, et non au bâtiment qui sert à son exposition. Néanmoins, on a jusqu'ici donné ce nom et à l'édifice et à la peinture. Le panorama consiste dans l'exécution d'un tableau qui offre les divers aspects de toute une contrée, telle qu'elle se développe dans la nature, sur l'entière circonférence de l'horizon, lorsque, placé dans un endroit élevé, on suit, en tournant la tête, l'ensemble des sites que l'œil peut embrasser. L'idée de tracer et de peindre ces aspects sur une toile circulaire, sans solution de continuité, est ce qui, avant tout, forma la base de cette application nouvelle de la peinture. Un mur également circulaire, ou rotonde propre à recevoir cette toile déroulée et exposée aux regards, ayant un plateau élevé au centre, devint donc la donnée principale de l'édifice. Mais une pareille peinture ne pouvant tirer son entier effet que du jour qui devait l'éclairer, sans qu'on pût en apercevoir la source, le progrès de l'invention consista, d'abord, à faire venir la lumière d'en haut, de manière que les rayons lumineux tombassent exclusivement sur le tableau ; ensuite à empêcher toute comparaison immédiate entre la lumière véritable et la lumière artificielle du dedans, la seule que l'artiste ait à sa disposition. Pour qu'on puisse obtenir ce résultat, l'arrivée dans l'intérieur doit avoir lieu au moyen de corridors entièrement obscurs. En détruisant ainsi peu à peu l'impression du jour naturel, on donnait à la lumière peinte l'apparence de la lumière réelle. Conduit mystérieusement sur le plateau central de la rotonde, le spectateur ne pouvait deviner la cause de la brillante clarté qui l'environnait. N'apercevant ni la limite inférieur ni le bord supérieur du tableau, cachés à sa vue, c'est-à-dire son commencement ni sa fin, il se perdait, pour ainsi dire, dans une immensité dont il ne pouvait se rendre compte. Sous l'influence des moyens matériels les plus ingénieux, joints aux ressources artielles [sic] les plus puissantes, pour donner à l'illusion l'aspect de la vérité, on conçoit comment, dans la représentation des

10 En 1881, Le Voltaire remarque : «Il y a trois ou quatre ans, Paris était pris de la fièvre du patinage à roulettes[...]. Maintenant nous entrons dans la Panoramanie. Cette fois nous sommes en plaine épidémie[...]. », cité dans F. Robichon, « Le panorama, spectacle de l'histoire », Le Mouvement social, 131, 1985, pp. 65-86. L'article de François Robichon donne des informations précieuses sur l'évolution du « marché » du panorama au dix-neuvième siècle, ainsi que sur le niveau de fréquentation et les goûts du public. Le genre disparaît brutalement, cependant, après l'Exposition universelle de 1900. Cf. également V.R. Schwartz, Spectacular Realities. Early Mass Culture in Fin-de-Siècle Paris, Berkeley, University of California Press, 1998. 
panoramas, l'application de ces moyens et de ces ressources peut produire les effets les plus merveilleux de la nature. ${ }^{11}$

La vogue du panorama s'installe de manière durable en Europe et aux Etats-Unis. La formule de la peinture circulaire enfermée dans une rotonde, que décrit Hittorff, est déclinée dans les directions les plus diverses. Une véritable mode des spectacles en rama s'empare des grandes métropoles européennes, où se multiplient les établissements qui proposent des spectacles du monde . Ainsi, à Paris ${ }^{12}$, on peut visiter, dans les années 18201830, outre les panoramas de Prévost, l'Alporama, l'Europorama, le Cosmorama, qui offre dans une galerie située au premier étage du Palais-Royal « la vue des monuments et sites les plus remarquables des quatre parties du Monde, tous les jours depuis 6 heures du soir jusqu'à onze ${ }^{13}$ », le Panstereorama, Porte maillot, qui expose « les modèles de Paris, Londres, Saint-Pétersbourg, Lyon, exécutés en relief sur une même échelle ${ }^{14}$ », l'Uranorama (Galerie Vivienne, représentation mobile du mouvement des astres), le panorama-voyageur de Mezzara, rue de Provence, le Peristrephorama ou Panorama nautique, l'Hydrorama, qui expose en 1829, dans un bateau, « un spectacle géographique et historique » constitué d'un petit panorama en demi-cercle, d'une chambre obscure et de vues d'optique, le Diaphanorama, le célèbre Diorama de Daguerre et Bouton, rue Samson depuis 1822, dont on adoptera le procédé à l'Opéra en 1825 pour le ballet de La Belle au bois dormant, le Néorama de Pierre Alaux, rue Saint-Fiacre, qui représente en 1827 l'intérieur de la Basilique saint Pierre de Rome et en 1827 l'Abbaye de Westminster à Londres ${ }^{15}$. On connaît l'écho ironique donné à cette « panoramanie », dans Le Père Goriot, par Balzac, qui avait été lui-même fortement impressionné par le diorama de Daguerre ${ }^{16}$.

11 J.J. Hittorff, « Description de la rotonde des panoramas, élevée dans les Champs-Elysées, précédée d'un aperçu historique sur l'origine des panoramas et sur les principales constructions auxquelles ils ont donné lieu », Revue générale de l'architecture et des travaux publics, vol. 2, 1841, $1^{\mathrm{er}}$ article, pp. 500-501.

12 Cf. G. Berthier de Sauvigny, Nouvelle histoire de Paris. La Restauration. 1815-1830, Paris, Hachette, 1977, pour une présentation des spectacles parisiens du temps.

13 Journal d'indications, 2 janvier 1810. Le cosmorama est transféré en 1828 rue Vivienne. Il est diffusé à New York (1815) et à Londres (1820).

14 Gazette de France, 26 juin 1810.

15 A. Gernsheim, L.J.M. Daguerre, New York, 1968, p. 43, ajoute à cette liste des dispositifs en rama que connaît le dix-neuvième siècle le Betaniorama, le Cyclorama, le Kalorama, le Kineorama, le Myriorama, le Nausorama, l'Octorama, le Physiorama, le Pleorama, le Poecilorama, le Typorama, l'Udorama. Cf. également R. Hyde, Panoramania. The art and entertainment of the 'all-embracing' view, Londres, Trefoil Publications, 1988. Mais le panorama va aussi donner son nom à des livres : ainsi le Panorama géographique français ou les mille et une beauté de la géographie de la France, publié à Paris en 1825, qui présente, pour chaque département, une carte, une vue des chefs-lieux, et une description des principales villes et curiosités.

16 «La récente invention du Diorama, qui portait l'illusion de l'optique à un plus haut degré que dans les Panoramas, avait amené dans quelques ateliers de peinture la plaisanterie de parler en rama, espèce de 


\section{3 - L’invention du géorama : Charles Delanglard}

En janvier 1829, le Prince Pückler-Muskau, aristocrate voyageur et raconteur d'histoires, commence sa visite des amas parisiens, ainsi qu'il les désigne (panorama, néorama, diorama, uranorama, cosmorama), par le « ama de la géographie, le géorama », au sujet duquel il affirme qu'aucun « globe ordinaire ne peut rendre la géographie si claire », souhaitant que des écoles s'installent dans de semblables «ventres de la terre ${ }^{17}$. Dans les divertissements nouveaux qu'offre la capitale, la géographie, à son tour, cherche à occuper une place, sous la forme très concrète d'un édifice : le géorama.

Le géorama est à l'origine, le pendant exact, pour ce qui concerne l'apprentissage de la géographie, des salles d'exposition à vocation panoramique et panoptique qui se répandent dans Paris sous la Restauration, puis la Monarchie de Juillet, et il s'inscrit, de la même manière que les panoramas, dans la configuration renouvelée des spectacles parisiens de cette époque. C'est, en effet, au $\mathrm{n}^{\circ} 7$, boulevard des Capucines, à proximité, par conséquent, des différentes rotondes qui avaient été élevées par Fulton, puis Thayer et Prévost, sur l'emplacement de l'ancien couvent des Capucines, qu'est installé, en 1825, le premier géorama, modèle pour les quelques réalisations postérieures que connaîtra le siècle. Son créateur est un employé à l'Administration Centrale des Contributions Indirectes, Charles Delanglard.

Celui-ci soumet à la Commission centrale de la Société de Géographie de Paris, dans sa séance du 17 mai 1822,

le projet d'une machine ronde ou globe, de 120 pieds environ [40 mètres] de circonférence, et représentant à l'œil la surface extérieure de la terre. Il la nomme Géorama. Il lit son mémoire et fait voir ses dessins. La Commission ordonne le dépôt de son Mémoire aux archives de la Société. ${ }^{18}$

Le texte du brevet déposé par Delanglard le 25 mars 1822 est plus précis dans la description du procédé, et permet de comprendre l'accueil intéressé qu'il a reçu dans un premier temps :

charge qu'un jeune peintre, habitué de la pension Vauquer, y avait inoculée. - Eh bien ! monsieurre Poiret, dit l'employé au Muséum, comment va cette petite santérama ?[...] - Il fait un fameux froitorama! dit Vautrin. [...] - Illustre monsieur Vautrin, dit Bianchon, pourquoi dites-vous froitorama? Il y a une faute, c'est froidorama. - Non, dit l'employé du Muséum, c'est froitorama, par la règle : j'ai froit aux pieds. », Balzac, Le Père Goriot (1834), Paris, Le Livre de Poche, 1995, p. 103-104.

17 H. Fürst Pückler-Muskau, Briefe eines Verstorbenen, Stuttgart, 1846, II, p. 409 et suivantes.

18 Bulletin de la Société de Géographie, t. I, 1822, p. 73. Parmi les membres de la Commission centrale, on trouve à cette date les noms de Barbié du Bocage, Letronne, Jomard, Walckenaer, Malte-Brun, Humboldt, Denon, Cuvier, Coquebert de Monbret, Champollion, Cuvier. 
Le Géorama est une machine à l'aide de laquelle on embrasse presque d'un seul coup d'œil toute la surface de la terre : il consiste en une sphère creuse de 40 pieds environ de diamètre [environ 13 mètres] au centre de laquelle le spectateur se trouve placé sur un plateau de 10 pieds environ de diamètre d'où il découvre toutes les parties du globe terrestre qui seront peintes à l'huile sur des châssis couverts de toile et qui tapissent exactement toutes les parois intérieures de la machine. Cette carte générale du monde sera dessinée d'après les meilleures cartes de géographie connues. ${ }^{19}$

A la différence des panoramas dont il est le contemporain, le géorama ne donne pas à voir un paysage, mais une carte, qui est peinte à l'intérieur d'une sphère. Il ne s'agit donc pas de faire le tour d'un globe artificiel, et de le considérer de l'extérieur, mais plutôt de se placer à l'intérieur du globe, sur une plate-forme, pour y regarder une mappemonde concave (illustration 1 : coupe du géorama, projet de 1822). Il ne s'agit pas non plus de voir la carte pour ainsi dire par-dessous et par transparence, comme si l'on était placé à l'intérieur d'une sphère dont la surface extérieure serait peinte. La précision donnée par Delanglard dans son Mémoire est importante, à cet égard, pour faire apparaître l'originalité de son projet :

Pour bien concevoir l'effet du Géorama, il ne faut point considérer cette machine comme représentant la forme de la terre, puisqu'il faudrait qu'elle offrît à l'œil du spectateur une surface convexe au lieu d'une surface concave. Il ne faut point non plus supposer le spectateur au centre de la terre qui serait diaphane et dont l'enveloppe extérieure, étant transparente, présenterait les terres et les mers vues par-dessous. Le Géorama présente la superficie de la terre et ne doit être considéré que comme une immense carte sans solution de continuité. 20

La rotonde conçue par Delanglard est assez analogue, d'un point de vue formel (en particulier pour ce qui concerne l'éclairage), avec le système mis en œuvre dans les panoramas de l'époque, même si, dans le cas du géorama, le spectateur se trouve non pas au centre d'une structure cylindrique, mais sphérique.

L'espace que doit occuper le pôle arctique au sommet de cette sphère est ouvert pour laisser pénétrer le jour dans l'intérieur à travers un vitrage de six pieds environ de diamètre. Le pôle opposé qui se trouve au bas est aussi occupé en partie par une cage d'escalier de 6 pieds environ de diamètre qui conduit au plateau - lequel est élevé de 15 pieds environ au-dessus du pôle antarctique, de sorte que l'œil du spectateur se trouve placé à la hauteur de l'équateur. Cet escalier

19 C. Delanglard, « Description du Géorama, son mécanisme et son usage », Brevet d'invention déposé le 25 mars 1822, n ${ }^{\circ} 1779$, agréé le 13 avril par le Comité consultatif des Arts et Manufactures, Ministère de l'Intérieur.

20 C. Delanglard, «Mémoire descriptif du Géorama », BNF, Cartes et Plans, Manuscrits de la Société de Géographie, Colis n 5, 1806 (souligné par Delanglard). 
est à jour tout au tour, en montant on peut donc voir toute la partie inférieure du globe. Autour du plateau règne un balcon circulaire auquel sont adaptées de longues baguettes mobiles qui servent d'index pour désigner plus facilement tel endroit que l'on veut; quelques verres grossissants seront aussi montés sur le bord de ce balcon afin que l'on puisse distinguer les petits détails qui pourraient échapper à l'œil ; des lampes placées dans la partie supérieure de la machine et d'autres autour de l'escalier, en dessous du plateau en rendront possible l'usage le soir, comme de jour.

Le géorama proprement dit, c'est-à-dire le globe, peut être accueilli et disposé, indifféremment, selon son concepteur, dans des édifices de formes et de natures variables. Delanglard propose néanmoins un édifice dont la construction effective aurait sans doute rendu le lieu assez attractif : il imagine de poser la sphère terrestre, réalisée alors en pierre, sur un support de dix colonnes, à l'imitation des temples antiques, ou révolutionnaires (illustration 2 : Elévation du géorama, projet de 1822). Dans son apparence extérieure, le géorama de Delanglard ressemble beaucoup aux temples conçus par Boullée, Ledoux et Lequeu à la fin du dix-huitième siècle (illustration 3 : Temple de la terre, J.-J. Lequeu, 1793).

Quant à la forme extérieure et à la construction du Géorama, elles peuvent varier suivant le local où il sera placé. Si on veut l'établir dans une salle de spectacle, dans une rotonde, ou dans tout autre vaisseau capable de le contenir, il pourra être construit en simples châssis de menuiserie ferrés et recouverts en toile ou en plâtre. Si on veut donner à cette machine une forme qui lui soit particulière, on peut l'exécuter comme elle est représentée sur les plans ci-joints et alors elle devra être construite en pierres, charpente, etc.

Le projet de géorama est accueilli favorablement. Delanglard parvient à louer un terrain boulevard des Capucines, à l'angle de la rue de la Paix ( $\left.{ }^{\circ} 30\right)$, sur lequel il construit l'édifice, qui ouvre ses portes en 1825. L'édifice est de taille modeste, plus proche des rotondes du boulevard Montmartre que de celles qui ont été réalisées dans le voisinage, boulevard des Capucines. La période de trois années nécessaire à l'exécution du projet a conduit, en outre, son concepteur à introduire quelques modifications. Un second « Brevet d'invention et de perfectionnement » est déposé en février $1825^{21}$. Delanglard transforme principalement trois éléments du géorama (illustration 4 : coupe du géorama, projet de 1825). Le plateau qui dans le premier projet était situé à hauteur de l'équateur, est désormais remplacé par une succession de trois galeries circulaires soutenues par des colonnes partant du rez-de-chaussée, munies de rampes métalliques, placées face à l'équateur et aux

21 Ministère de l'Intérieur, Administration générale des Haras, de l'Agriculture, des Manufactures, du Commerce, etc., Bureau des Manufactures, $n^{\circ}$ 2555. Certificat délivré le 31 mars 1825. 
tropiques, et auxquelles le spectateur accède par un escalier à double révolution. Delanglard présente ainsi la seconde modification :

Sur une partie des carreaux résultant de l'assemblage des tringles de fer est tendu un tissu transparent dont l'effet est de représenter les mers qui couvrent le globe, les autres carreaux sont remplis par un corps opaque sur lequel est fixée la carte qui présente les continents et les îles. [...] La première sphère était opaque et composée de châssis peints à l'huile, de sorte que le jour n'aurait eu accès dans l'intérieur que par une ouverture de six pieds environ, pratiquée au sommet, tandis que dans la nouvelle, il pénètre par toutes les mers qui sont en tissu très fin et transparent. La carte est maintenant peinte à 1 'aquarelle. ${ }^{22}$

La troisième transformation concerne le bâtiment lui-même, auquel Delanglard, semble-t-il, ne parvient pas à donner l'apparence initialement prévue. En témoigne la description insérée dans la notice de présentation, probablement rédigée par celui qui est désormais le directeur du géorama, et diffusée à l'occasion de l'ouverture de l'établissement :

Le Géorama offre à l'œil le spectacle imposant de l'ensemble du globe terrestre, représenté dans l'intérieur d'une sphère de grande dimension. Le monument qui renferme cette sphère colossale est un octogone éclairé de tous côtés, et dont la façade est ornée d'un péristyle supporté par quatre colonnes d'ordre dorique composite. A la suite est un vestibule qui précède une rotonde, dont les plafonds transparents et richement peints jettent un jour mystérieux sur cette enceinte élégamment décorée, et du milieu de laquelle s'élèvent, entre trente-deux colonnes arabesques, deux escaliers en spirale, qui conduisent à trois étages de galeries circulaires. Là les spectateurs isolés au milieu d'un globe de 120 pieds de circonférence, distinguent les principales villes, les fleuves, les rivières, les lacs, les montagnes, les volcans, les mers et les îles du monde entier. ${ }^{23}$

La sphère n'est donc pas exposée à l'air libre, offerte aux regards de la rue, comme le projet initial l'avait envisagé. Elle est désormais enfermée dans un bâtiment, dont Delanglard avait auparavant pris soin de signaler qu'il serait percé « de plusieurs croisées tant sur ses divers côtés que sur son toit». A l'instar des panoramas, le géorama donne le

22 C. Delanglard, «Description de la machine dite Géorama inventée et perfectionnée par Charles François Paul Delanglard», pièce jointe à la « demande de Brevet d'invention et de perfectionnement pour dix années » déposée le 2 février 1825.

23 J.G.V. de Moléon, Annales mensuelles de l'industrie manufacturière, agricole et commerciale..., Paris, 1827, t. II, « Bulletin des établissements utiles », p. 219. Il n'est pas impossible, comme le remarque R. Altick (The Shows of London, Cambridge et Londres, Harvard University Press, 1978, p. 464), que la structure intérieure, mais aussi, peut-on ajouter, l'apparence extérieure, du Colosseum de Londres, ait été inspirées par le Géorama. 
monde à voir dans une pénombre qui renforce le caractère d'illusion et de mystère du dispositif adopté.

A vrai dire, on peut considérer l'édifice imaginé par Charles Delanglard dans la première version de son projet comme l'agrandissement, certes spectaculaire et porté jusqu'à l'échelle de l'espace urbain, des globes terrestres utilisés depuis le seizième siècle, comme instruments d'étude, dans les apprentissages géographiques ${ }^{24}$. Bien souvent, en effet, ces petits globes artificiels sont supportés eux-mêmes par une structure composée d'une colonnade, analogue à celle qui est envisagée par Delanglard. La grande différence, cependant, entre le système du géorama d'avec ces globes, ainsi d'ailleurs d'avec les dispositifs qui seront mis en place, par exemple, en 1875, à la Bibliothèque Nationale, pour exposer au public les grands globes de Coronelli, tient dans le fait que, pour ce qui concerne ces derniers, les spectateurs restent autour du globe, tandis que dans le cas du géorama, comme on l'a déjà dit, ils y pénètrent (illustration 5 : exposition des globes de Coronelli en 1875). Delanglard y voit un avantage pédagogique considérable. L'objectif avoué de sa «machine » est, en effet, de pallier les défauts usuels de la carte plane, mais aussi de la sphère convexe :

Quoique le Géorama présente l'inverse de la réalité, il a un avantage marqué sur les cartes planes qui n'offrent que des fragments du globe, et sur les sphères convexes qui, lorsqu'elles sont volumineuses surtout, ne présentent à l'œil que le seul point qui en est le plus rapproché.

Ni la mappemonde, ni la sphère convexe, ne permettent de saisir la totalité de la surface de la terre d'un seul coup d'œil. La concavité de la mappemonde placée à l'intérieur d'une sphère doit, à l'inverse, rendre possible un tel regard.

Le projet de Delanglard doit, en réalité, être rapporté au contexte d'un problème ancien dans l'histoire de la géographie, constitutif en un sens de l'intention cartographique elle-même. En effet, si la mappemonde est nécessaire, dit Ptolémée, c'est parce que « la terre véritable, qui est de très grandes dimensions et ne nous entoure pas, ne peut être parcourue en entier par un même individu 25 ». La représentation géographique a pour raison d'être de procurer cette vision globale, immédiate, de la surface de la terre, vision qui reste matériellement inaccessible à l'œil naturel du spectateur. Mais, comme Delanglard le

24 F. de Dainville, «Les amateurs de globe», Gazette des Beaux-Arts, t. LXXI, janvier 1968, pp. 51-64. M. Pelletier, « De l'objet de luxe au produit de consommation courante. L'évolution de l'édition des globes en France, aux XVIII ${ }^{\mathrm{e}}$ et $\mathrm{XIX}^{\mathrm{e}}$ siècles », dans Tours et contours de la terre, Paris, Presses de l'Ecole Nationale des Ponts et Chaussées, 1999, pp. 47-56.

C. Ptolémée, Géographie, I, I, 6. 
souligne, le géorama est plus qu'une mappemonde. Il va plus loin que la mappemonde et le globe convexe, tout en permettant d'en accomplir véritablement l'intention. Pour comprendre cette affirmation, il faut encore solliciter Ptolémée. Celui-ci compare la géographie avec l'astronomie, pour signaler l'avantage que possède cette dernière : « les mathématiques permettent d'expliquer à l'intelligence humaine le ciel lui-même tel qu'il est au naturel, car on peut le voir tourner autour de nous ${ }^{26} »$. Avec l'astronomie donc, telle que Ptolémée la présente, nul besoin de ce genre d'intermédiaire symbolique que constitue la carte : l'œil, certes exercé par les mathématiques, suffit. A la différence de la surface de la terre, qui ne se découvre aux regards du géographe que par pièces successives, le ciel se donne à voir à l'astronome dans sa totalité. Tout se passe, finalement, comme si le projet de Delanglard était une application de cette remarque de Ptolémée. Si le géorama l'emporte, du point de vue de son efficacité représentative, sur la mappemonde et le globe convexe, s'il permet effectivement de saisir d'un coup d'œil synthétique la totalité ou presque de la surface de la terre, c'est parce que, en un certain sens, dans le géorama la terre est devenue un ciel $^{27}$. Tout comme dans les panoramas qui sont réalisés à la même époque (et dans le même quartier de Paris, pourrait-on ajouter), le spectateur placé au centre du géorama est quasiment enveloppé par la réalité qu'il découvre sans solution de continuité. Il est dans l'illusion perceptive de la totalité terrestre, qui l'entoure.

Dès le mois de mai 1825, Edme Jomard, l'un des fondateurs de la Société de géographie de Paris et du Cabinet de géographie de la Bibliothèque Nationale, ancien membre de la commission et de l'Institut d'Egypte, peut annoncer dans le Bulletin de la Société :

Il vient de se former à Paris, sous le nom de Géorama, un établissement d'un genre tout nouveau : c'est l'exécution du projet que M. Delanglart soumit à la Société, dans le courant du mois de mai 1822. Introduit par deux escaliers circulaires qui s'élèvent du pôle austral vers le pôle arctique, autour de l'axe terrestre, dans un globe de cent-vingt pieds de circonférence, et qui n'est éclairé que par la matière transparente dont il est formé, le spectateur découvre, sur son ample circonférence, les différentes régions de la terre ; porté sur trois galeries qui forment trois étages, et qui soutenues ellesmêmes par le double escalier, sont entièrement isolées du globe, il fixe à volonté son attention sur le point qu'il préfère ; sa vue domine partout avec avantage. Il peut passer successivement à toutes les

26 Ibid.

27 «... en jouissant de ce spectacle, on est placé sous la voûte du globe; on ne circule plus autour de sa surface... », indique le Secrétaire Général de la Société de géographie, Roux, lors de l'Assemblée générale du 25 novembre 1825 (Bulletin de la Société de Géographie, t. IV, p. 275). 
parties et dans toutes les directions. Il n'existe aucune interruption : c'est dans les lignes des méridiens et des parallèles qu'on a ménagé les jointures indispensables. L'œil embrasse facilement à la fois la plus grande partie de l'ensemble. Ce tableau sphérique n'est au globe terrestre que dans la proportion de 1 à 1000000 ; mais la magie de la peinture et de l'éclairage, projetant au loin les objets, leur donne des dimensions beaucoup plus considérables. Cet établissement servira particulièrement à l'étude de l'ensemble des masses, et l'éclat, pour ainsi dire, populaire, que la géographie doit en recevoir, ne pourra qu'en propager le goût. ${ }^{28}$

Désormais soutenu par un tel patronage, le géorama voit alors les visites se succéder. Les témoignages dont on dispose évoquent les passages de plusieurs membres de la Société de géographie, de militaires, de navigateurs et de géographes, dont Jomard luimême et Alexandre de Humboldt. On a déjà fait allusion au passage de Pückler-Muskau. Le journal anglais The Literary Gazette souligne, en octobre 1827, que le géorama « is one of the most pleasing exhibitions of the French capital, and is remarkable, not to say unique, for the degree in which it combines the advantage of scientific instruction with the gratification afforded by a grand and imposing spectacle ${ }^{29} »$. Le géorama reçoit également quelques visiteurs de marque : la duchesse de Berry, la duchesse et le duc d'Orléans (futur LouisPhilippe), le jeune duc de Bordeaux (petit-fils du futur Charles X). L'efficacité pédagogique du procédé inventé par Delanglard conduit à quelques démarches ministérielles, restées sans suite, en vue de « faire du géorama le siège et le foyer d'un enseignement géographique ${ }^{30}$ ».

\section{4 - Géorama et vue d'ensemble : Alexandre de Humboldt}

Un des membres de la Société de géographie les mieux disposés à l'égard du géorama est sans doute Alexandre de Humboldt, qui y retrouve peut-être une idée qui lui est familière. Jullien, fervent défenseur de Delanglard, en témoigne ainsi :

M. de Humboldt, juge très compétent, avec lequel je visitai le géorama, il y a environ 2 ou 3 ans, trouva bonne et ingénieuse l'idée de placer le spectateur dans une situation telle qu'il pût embrasser d'un seul coup d'œil toutes les contrées du globe et l'immensité des mers et rapprocher et comparer les différents pays, par une sorte d'intuition immédiate. Il voulut bien indiquer plusieurs améliorations dont le géorama lui semblait susceptible, et exprimer le vœu qu'on

E.F. Jomard, Bulletin de la Société de Géographie, Première série, t. III, Paris, Arthus Bertrand, $\mathrm{n}^{\circ} \mathrm{XXV}$, mai 1825 , p. 289.

The Literary Gazette, and Journal of the Belles Lettres, 20 octobre 1827, p. 685.

30 Lettre de M. Jullien au Président de la Commission centrale de la Société de géographie, 18 décembre 1829 (BNF, Cartes et Plans, Manuscrits de la Société de Géographie, Colis $n^{\circ} 19$ bis, 3282). Le ministre concerné est Vatimesnil. 
pût y établir une école centrale de géographie, non seulement pour les jeunes gens des deux sexes, et pour les élèves des collèges royaux et des nombreuses institutions de la capitale, mais aussi pour les hommes du monde dont la plupart ont à refaire entièrement leur éducation géographique, si défectueuse et incomplète jusqu'ici dans nos établissements universitaires et dans les meilleures maisons d'éducation. ${ }^{31}$

La raison de l'intérêt de Humboldt pour le procédé de Delanglard va plus loin que le simple, et récurrent, argument pédagogique. Les termes mêmes qui sont utilisés par le témoin qui rapporte ses propos indiquent que le géorama, en fait, s'accorde avec la conception de la géographie, et plus largement de la science, que défend le géographe allemand. Humboldt a souligné à plusieurs reprises, en effet, tout en regrettant qu'ils fussent plutôt dédiés à la représentation des villes qu'à celle des « grandes scènes dans lesquelles la nature étale sa sauvage abondance et toute la plénitude de la vie», les services que les dispositifs artistiques en rama pouvaient rendre à la représentation scientifique du monde. Il leur attribue, dans un premier temps, deux vertus corrélatives. La longue page qu'il consacre à la question mérite d'être lue en entier :

Malgré l'état peu satisfaisant où sont demeurées jusqu'ici les gravures qui accompagnent et souvent déparent nos relations de voyage, elles n'ont pas peu contribué cependant à faire connaître la physionomie des zones lointaines, à répandre le goût des voyages dans les contrées tropicales, et à stimuler activement l'étude de la nature. Les décors de théâtre, les panoramas, les dioramas, les néoramas, et toute cette peinture à grande dimension, si fort perfectionnée de nos jours, ont rendu plus générale et plus forte l'impression produite par le paysage. Vitruve et le grammairien Jules Pollux nous ont décrit les décorations champêtres qui servaient à la représentation des pièces satyriques. Longtemps après, vers le milieu du $\mathrm{XVI}^{\mathrm{e}}$ siècle, l'établissement des coulisses, dû à Serlio, favorisa beaucoup l'illusion; mais aujourd'hui, après les admirables perfectionnements apportés par Prévost et Daguerre à la peinture circulaire de Parker, on peut presque se dispenser de voyager à travers les climats lointains. Les panoramas circulaires rendent plus de services que les décors de théâtre, parce que le spectateur, frappé d'enchantement au milieu d'un cercle magique, et à l'abri de distractions importunes, se croit entouré de tout côté par une nature étrangère. Ils nous laissent des souvenirs qui, après quelques années, se confondent avec l'impression des scènes de la nature que nous avons pu voir réellement. Jusqu'à présent, les panoramas, qui ne peuvent faire illusion qu'à la condition d'avoir un large diamètre, ont représenté des villes et des lieux habités, plutôt que les grandes scènes dans lesquelles la nature étale sa sauvage abondance et toute

31 Ibid. 
la plénitude de la vie. Des études caractéristiques prises sur les flancs escarpés de l'Himalaya et des Cordillères, ou au milieu des fleuves qui sillonnent les contrées intérieures de l'Inde et de l'Amérique méridionale, produiraient un effet magique, si l'on avait soin surtout de les rectifier d'après des empreintes prises au daguerréotype, excellent pour reproduire, non pas les massifs de feuillage, mais les troncs gigantesques des arbres et la direction des rameaux. ${ }^{32}$

Les panoramas, ainsi que les dispositifs circulaires du même genre, possèdent une première vertu, qu'ils partagent avec l'ensemble des instruments de représentation géographique : ils semblent pouvoir se substituer au voyage, parce qu'en eux-mêmes ils se présentent comme d'authentiques voyages d'imagination. Mais ils possèdent en outre une seconde vertu, qui vient renforcer la première et lui donner son ampleur véritable. Car ces voyages, grâce aux dispositifs panoramiques, sont comme de véritables transports de l'imagination et, surtout, de la sensibilité. L'ombre au sein de laquelle le spectateur progresse vers la plate-forme, la lumière zénithale sur la toile, l'absence de cadre et de «solution de continuité » (Hittorff) dans la peinture et la scénographie, induisent le spectateur à se sentir comme enveloppé par le spectacle qu'il contemple, et, alors, placé dans un « cercle magique », il fait l'expérience sensible d'une « étrangeté » en tout point analogue à celle qu'il pourrait (ou a pu) éprouver au contact d'une réalité lointaine et d'une nature vraiment étrangère. L' « enchantement» dont parle Humboldt est celui d'un voyage d'initiation, mais d'initiation au monde, sans qu'il soit possible de décider si ce voyage est réel ou bien imaginaire, sans, d'ailleurs, qu'il soit nécessaire, peut-être, de décider. D’une façon qui n'est paradoxale qu'en apparence, l'illusion qui se déploie dans le panorama plonge le spectateur dans l'épreuve de la réalité, et l'égarement de l'esprit qui saisit le spectateur quant à l'impression magique et à la puissance de réalité qu'il éprouve, est analogue à celui du voyageur qui rencontre, comme Alexandre de Humboldt peut lui-même en témoigner, un univers exotique. L'imagination, poussée ainsi à son maximum, possède autant de pouvoir sur l'esprit que l'inimaginable. Et l'étrangeté de l'expérience qui se vit dans les rotondes panoramiques, au cœur des grandes villes, fait résonner, dans l'âme du spectateur, l'étrangeté et la puissance du monde, son « ailleurs », tel qu'il pourrait aussi le vivre, s'il était voyageur.

Cependant, si les dispositifs panoramiques éduquent le spectateur dans le sens du développement de sa sensibilité, ils possèdent également une autre propriété, fondamentale, grâce à laquelle, à la sensibilité au monde, s'adjoint la connaissance du monde.

32 A. de Humboldt, Cosmos, t. II, Paris, 1855, pp. 105-106. 
Tous ces moyens, dont nous ne pouvions manquer de faire l'énumération dans un livre tel que le Cosmos, sont très propres à propager l'étude de la nature; et sans doute la grandeur sublime de la création serait mieux connue et mieux sentie, si dans les grandes villes, auprès des musées, on ouvrait librement à la population des panoramas où des tableaux circulaires représenteraient, en se succédant, des paysages empruntés à des degrés différents de longitude et de latitude. C'est en multipliant les moyens à l'aide desquels on reproduit, sous des images saisissantes, l'ensemble des phénomènes naturels, que l'on peut familiariser les hommes avec l'unité du monde et leur faire sentir plus vivement le concert harmonieux de la nature. ${ }^{33}$

Plus que l'ambiance «physico-théologique » du vocabulaire, ce qu'il faut retenir dans cette remarque de Humboldt (qui reprend l'idée de Miel sur la dissémination des panoramas dans les grandes villes) au sujet de la succession des tableaux, c'est la volonté de réunir les conditions d'une vue synoptique (ce qu'il appelle une « vue d'ensemble ») du monde terrestre, et par là de sa connaissance générale. Il faut considérer ce défilé de paysages appartenant à des régions différentes du monde comme un travelling intelligent. La vitesse de la succession est la condition de la clarté de la comparaison, et, au-delà, elle est la clé d'accès à la saisie des ordres généraux de l'espace terrestre. Méthode géographique, la comparaison des caractères des régions, qui fait apparaître les parentés et les différences, les continuités et les ruptures, c'est-à-dire les entités géographiques (qu'elles soient naturelles ou humaines), n'est rien d'autre que la mise en œuvre d'un raisonnement spatial. Et le géorama, qui permet au spectateur d' « embrasser d'un seul coup d'œil toutes les contrées du globe et l'immensité des mers et rapprocher et comparer les différents pays, par une sorte d'intuition immédiate », est un des supports privilégiés, malgré son caractère statique, de cette éducation géographique selon Humboldt.

La vertu centrale des dispositifs panoramiques, dont le géorama est une espèce, est donc une vertu synoptique, et même panoptique, comme le relève fort justement Bernard Comment : le monde s'offre au sujet, en position centrale, « sur le mode du Rundblick, ce regard continu qui embrasse tout l'horizon d'un seul coup ou presque ${ }^{34} »$. Regardant la carte depuis, pour ainsi dire, le centre de la terre, dont la surface l'entoure comme une voûte céleste, le spectateur est comme placé au-dessus du monde, pouvant alors y accéder d'un

33 Ibid., pp. 106-107.

34 B. Comment, op. cit., p. 96. Même formulation dans la description d'un des géoramas qui prolonge celui de Delanglard, en 1844, à propos de la concavité de la représentation : « cette concavité a l'avantage de laisser voir tout le développement du sphérö̈de, en permettant au spectateur d'embrasser d'un coup d'œil les parties les plus opposées, et d'en saisir tous les rapports » (S. Berthelot, « Notice sur le Géorama de M. Ch. A. Guérin... », Bulletin de la Société de géographie, $3^{\mathrm{eme}}$ série, t. II, 1844, p. 99). 
seul regard ${ }^{35}$. Mais ce panoptisme est également ce qui caractérise essentiellement le projet intellectuel de Humboldt, tel que celui-ci l'exprime lui-même dans une lettre à Varnhagen von Ense, à propos du Cosmos :

Je commence l'impression de mon ouvrage, de l'œuvre de ma vie entière. Tout le monde matériel, tout ce que nous savons aujourd'hui des phénomènes des cieux et de la terre, depuis les nébuleuses jusqu'à la géographie de la mousse qui croît sur les rochers de granit, voilà ce que j'ai l'extravagante pensée de concentrer dans un seul ouvrage qui, pour la vivacité de la forme, captive l'esprit et le cœur. Toute grande et importante idée, qui a rayonné quelque part, doit y avoir sa place à côté des faits. ${ }^{36}$

Le livre-monde de Humboldt est, d'une certaine manière, l'héritier, et tout à la fois le résumé des atlas de la Renaissance, des décors et des jardins géographiques, ainsi que des géoramas qu'il a lui-même visités ${ }^{37}$. Il en donne la formule, et en exprime l'intention. Lorsque Paul Vidal de la Blache, à son tour, tentera d'esquisser le programme fondamental de la géographie humaine, il retrouvera l'intuition humboldtienne, à laquelle il fera référence :

L'idée qui plane sur tous les progrès de la géographie est celle de l'unité terrestre. ${ }^{38}$

La géographie est fondée, ajoute le géographe français, sur « la conception de la terre comme un tout dont les parties sont coordonnées, où les phénomènes s'enchaînent et obéissent à des lois générales 39 ». Et le géorama peut, sans doute, être considéré comme une des préfigurations de ces musées que Vidal de la Blache appelle de ses vœux, où « l'homme peut s'étudier lui-même, non point in abstracto, mais sur des réalités ».

35 M.-A. Jullien, un des plus ardents défenseurs de Delanglard à la Société de Géographie, publie en 1845 un court texte dont le titre prolonge cette atmosphère «humboldtienne »: Géorama, ou vue intuitive du globe terrestre, Paris. Jullien se présente dans ce texte comme un des fondateurs du géorama de Delanglard. Lettres de Humboldt à Varnhagen von Ense, Genève, 1960, p. 15 (lettre du 24 octobre 1834), 